\title{
El microcrédito, elemento clave del desarrollo económico rural: un estudio de caso
}

\author{
Microcredit, a key element of rural economic development: a case study
}

\author{
Mario Orozco-Gutierrez \\ Doctor en Gestión para el Desarrollo, Universidad Autónoma de Chiapas, \\ Tuxtla Gutiérrez - México, mogli_swan@hotmail.com
}

\begin{abstract}
Cómo citar / How to cite
Orozco-Gutierrez, M. (2019). El microcrédito, elemento clave del desarrollo económico rural: un estudio de caso. Revista CEA, 5(9), 147-159. https://doi.org/10.22430/24223182.1313
\end{abstract}

Recibido: 16 de octubre de 2018

Aceptado: 27 de diciembre de 2018

\begin{abstract}
Resumen
Para las personas que carecen de educación financiera es complicado iniciar un negocio. Por no tener acceso a la banca comercial, para conseguir financiamiento recurren a los amigos, familiares y prestamistas. Actualmente, las microfinanzas suplen estas formas de obtención de dinero y este impulso económico hace que, especialmente las mujeres, puedan invertir, permitiéndoles atenuar los gastos en sus hogares. Este tipo de microfinanciamiento está impactando económicamente a las comunidades rurales; los resultados indican que se han conformado grupos de mujeres emprendedoras, generando un aumento importante en la economía del lugar. El propósito del estudio, de tipo cuantitativo y descriptivo, es identificar las características de quienes solicitan microcréditos; esto en una muestra de 50 emprendedores. Los datos obtenidos revelan que, en 2016, se destinó un promedio de 50.000 micropréstamos en la costa de Chiapas, de ellos el $70 \%$ se destina como capital de trabajo, y el $30 \%$ restante como activo fijo.
\end{abstract}

Palabras clave: pequeñas empresas, microfinanciamiento, modelo de microfinanciamiento, desarrollo económico rural.

Abstract
Individuals who lack financial education have a hard time starting a business. As they have no access
to commercial banking, they turn to friends, family, and moneylenders. Currently, microfinance
satisfies those money needs and its economic boost enables individuals, especially women, to invest
and reduce their household expenses. Microfinance is also impacting the economy of rural
communities. The results indicate that groups of entrepreneurial women have been formed,
producing a significant growth of their local economy. This quantitative descriptive study aims at
identifying the characteristics of those who apply for microcredits by analyzing a sample of 50
entrepreneurs. The data obtained in this work reveal that, in 2016, an average of 50,000 microloans 
were granted in the coast of Chiapas; $70 \%$ of them were devoted to working capital and the remaining $30 \%$, to fixed assets.

Keywords: small enterprises, microfinancing, microfinancing model, rural economic development.

\section{INTRODUCCIÓN}

Entre las políticas de desarrollo para la población mundial, la Organización de Naciones Unidas (ONU) ha establecido como uno de sus más importantes objetivos la reducción de la pobreza, y reconoce como uno de los elementos que puede facilitar su cumplimiento el otorgamiento masivo de operaciones de microcrédito, motivo por el cual declaró al año 2005 como el Año Internacional del Microcrédito (Aguilar-Pinto, Tuñón-Pablos, \& y Morales-Barragán, 2017). La ONU instó a los gobiernos a generar programas de microcréditos para disminuir la pobreza, fomentar el trabajo por cuenta propia y generar ingresos.

La Cumbre del Microcrédito sostiene que 14.2 millones de las mujeres más pobres del mundo, actualmente tienen acceso a servicios financieros en instituciones microfinancieras (IMF) especializadas, bancos, Organizaciones No Gubernamentales (ONG) y otras instituciones financieras bancarias (Cheston \& Kunh, 2008). La mayoría de estas mujeres tienen acceso al crédito para invertir en negocios propios que ellas mismas operan; asimismo, tienen excelentes registros de pagos, a pesar de las carencias diarias que enfrentan (Cruz Ramirez, Pérez Castañeda, \& Piedra Mayorga, 2013).

Según el Banco Mundial, la sociedad en general debe concentrar su atención en mejorar el empoderamiento económico y el aumento de las inversiones hacia las mujeres, pues la mayor participación de ellas en la fuerza de trabajo da como resultado mayores ganancias y reducción de la pobreza; las mujeres se benefician de un mayor empoderamiento, así como los hombres, los niños y la sociedad en general (Girón, 2014).

En México, el Consejo Nacional de Evaluación de la Política de Desarrollo Social-CONEVAL-asume la premisa de que la estrategia más eficiente que permite afrontar la pobreza «en forma definitiva» es el uso de las microfinanzas, haciendo crecer la economía, crear empleos, impulsar políticas sociales, los servicios de salud, vivienda y la educación, no solamente la profesional sino dándole importancia a la educación financiera (Cruz Ramirez, et al., 2013).

Según los analistas internacionales, el microcrédito debe ser una herramienta emancipadora y determinante para superar el círculo de la pobreza En la actualidad se observa que 70 de cada 100 habitantes en condiciones de extrema pobreza urbana son mujeres, pero tienen un amplio potencial de generar microempresas, por lo que se debe promover la entrada a los mercados y permitirles los mecanismos de financiamiento que requieren las mujeres empresarias, para ingresar en la pequeña y mediana industria (Heller, 2010).

Es importante mencionar que cuando los microcréditos son manejados por mujeres, se observa un alto grado de responsabilidad en la administración de los recursos debido a que casi la totalidad de 
los microfinanciamientos otorgados son recuperados en los tiempos establecidos, en un porcentaje superior al $98 \%$. (Cruz Ramirez, et al., 2013).

En México, al igual que en todo el mundo, el modelo de microfinanciamiento ha sido utilizado como una alternativa para ampliar la capacidad empresarial y para salir de la pobreza. Con base en la Conferencia Internacional sobre Microcrédito, en 1997, estos consisten en programas de concesión de pequeños créditos a los individuos que alcanzan los más altos índices de pobreza, para que puedan poner en marcha pequeños negocios que generen ingresos que mejoren su nivel de vida y el de sus familias (Patiño, 2008).

Actualmente, la premisa para el crecimiento económico y el desarrollo sustentable del microempresario individual ha sido acompañada de una antipatía implícita, como una forma de intervención estatal ante el fracaso de la banca de desarrollo (Cabezas, 2008) y la intermediación financiera, ya que las altas tasas de interés que se cobran (Esquivel, 2010) son expresión del proceso de financiamiento existente, producto de los inversionistas (Bateman \& Chang, 2012; Girón, 2014) y "como una condición para abatir la pobreza», donde se ponen de manifiesto las fallas de mercado, que han dado como resultado mercados de crédito fuera del sistema financiero (Armendáriz y Morduch, 2011).

Dentro de las políticas públicas elaborada por el gobierno de México, se han creado varios programas de apoyo, que van dirigidos especialmente a grupos de población con más escasos recursos económicos como el Programa Nacional de Financiamiento al Microempresario (PRONAFIM), que otorga a los núcleos familiares y a personas de más bajos recursos pequeños préstamos conocidos como microcréditos, con la finalidad de apoyarlos económicamente en sus actividades productivas, para que puedan producir pequeños negocios y puedan generar autoempleo (PRONAFIM, 2013).

Debido a esto, la mayoría de los microcréditos se enfocan a las comunidades rurales por ser más vulnerables y pobres, porque son personas no asalariadas que carecen de alguna garantía, pero los préstamos otorgados son de menor cuantía. Para que las personas puedan obtener un nuevo crédito deben pagarlo en su totalidad en un término de 15 semanas, para que así se les pueda otorgar un nuevo préstamo y mantener "su derecho a ser sujetos de crédito», por lo que la clientela está formada en su mayoría por mujeres (Banco Compartamos, 2016).

Como contraparte al PRONAFIM, surge en 1999 otro programa similar que está dirigido al sector rural, exclusivamente a mujeres: el programa FOMMUR (Fondo de Microfinanciamiento a Mujeres Rurales) derivado del programa PRONAFIM, con el propósito de impulsar el autoempleo y las actividades y/o prácticas productivas, así como las habilidades empresariales básicas de fomentar el ahorro y generar ingresos entre las mujeres de escasos recursos del medio rural, marginadas o no marginadas, con proyectos productivos viables de ser financiados; el objetivo del establecimiento de este programa es dotar de un mecanismo de microfinanciamiento que permita proveer dinero en forma de créditos simples y sencillos a mujeres del sector rural, que son personas que por su origen no cuentan con acceso a los servicios financieros de la banca comercial (Gobierno de México, 2012).

EI PRONAFIM afirma que "debido a esto, el $85 \%$ de los microcréditos va dirigido a las mujeres, quienes por lo general instalan micro negocios relacionados con la cocina, la costura, manualidades, venta de ropa, cosméticos, productos de limpieza, comercialización de hortalizas y alimentos, 
abarrotes y artesanías entre otros». Sin embargo, existe una tendencia contradictoria, las microempresas comandadas por mujeres son por lo general menos rentables, producto de la orientación de los microcréditos dirigidos a dar una continuidad al papel tradicional en el desempeño de las labores de la mujer: elaboración y venta de ropa, alimentos, comercio al por menor, servicios en pequeña escala (Cabezas, 2008).

Uno de los motivos más aceptados, por la cual las Instituciones microfinancieras deben comprometerse a incursionar en las zonas rurales de México es porque, dentro de ellas, la pobreza y marginación es más elevada que en la porción urbana. De esta manera, las mujeres constituyen los grupos más vulnerables dentro de ese sector, de ahí que sean ellas a quienes van dirigidos los microcréditos (Cheston \& Kuhn, 2008). Por otra parte, la cohesión social que hay entre las pequeñas comunidades permite con mayor facilidad la aplicación de la metodología y el enfoque de las microfinanzas.

Actualmente se proporciona a las mujeres servicios financieros por su capacidad innovadora y su habilidad de beneficiarse de este acceso a los microcréditos, limitada únicamente por las desventajas del género. Por ejemplo, la gran diferencia en la cantidad de los préstamos puede indicar que hay una discriminación social hacia las mujeres, esta situación limita las oportunidades disponibles para ellas; esto es importante ya que esto permite valorar los programas de desarrollo que utilizan a los microcréditos como palanca para el combate a la pobreza (Cruz Ramirez, et al., 2013).

Según lo anterior, el objetivo del estudio se centra en realizar un perfil de las personas emprendedoras en relación con el acceso a los microcréditos e identificar si estos inciden en el desarrollo rural en la comunidad de Paredón, Chiapas, con la finalidad de propiciar el empoderamiento y las oportunidades económicas para las mujeres en este lugar, con una muestra de 50 microempresarios; aquellos que han utilizado micropréstamos para iniciar sus negocios.

\section{MARCO TEÓRICO}

\section{Los microcréditos en Chiapas}

Los autores Armendáriz y Morduch (2011), en su libro Economía de las Microfinanzas privilegian las dimensiones del alcance de la población atendida por las microfinanzas, ya que hacen mención especial en la discusión que plantean sobre la amplia participación de las mujeres sobre la significancia empírica de tal participación, el potencial de estas para cambiar las normas sociales para las mujeres y el efecto de esta participación en la eficiencia de las instituciones, donde se habla de la alta responsabilidad de las mujeres, principalmente en la forma de los pagos de las deudas contraídas. Más allá del tema del género, es innovadora la discusión de los autores sobre los efectos de las microfinanzas, al incluir recientes temas de discusión sobre sesgos de selección y posibles aspectos y métodos de evaluación de impacto entre los clientes.

De acuerdo con la literatura sobre los microcréditos, las entidades microfinancieras son parte del sistema financiero oculto, al sustituir el financiamiento que el Estado debería de proporcionar, con base en sus políticas de desarrollo, con el propósito de disminuir las desigualdades sociales y que consiste en transformar a las mujeres en agentes económicos, en seres con «libertad para elegir», 
pero no solo para poder determinar el uso del crédito e insertarse en proyectos productivos, sino como emprendedoras en las decisiones administrativas, políticas y sociales de la sociedad (Girón, 2014).

Chiapas es el estado mexicano que cuenta con el mayor número de entidades microfinancieras actualmente hay 132, lo contrario del norte de la República, que hay muy pocas; en Baja California sur hay únicamente 1, Durango 1, Colima 2, Zacatecas 3 y Baja California 5 (Cruz Ramirez et al., 2013). En la entidad el sector microfinanciero ha operado una política sumamente agresiva, promoviendo tasas de interés elevadísimas, buró de crédito ineficaz, tecnología incipiente, y-lo más serio- clientela compartida (entre $30 \%$ y 50 \% participan en dos o más instituciones), en particular, las principales microfinancieras que operan son: Compartamos, Micronegocios Azteca y Te Creemos (El Economista, 2013).

En estos lugares de la zona Istmo-Costa de Chiapas, la gente pobre utiliza los microcréditos para hacer mejoras a sus casas, principalmente para hacer techos de lámina y pisos de cemento; las mujeres emprendedoras lo utilizan para compras de bienes suntuarios, pero principalmente les ha servido para capitalizar sus micronegocios, en la compra de mercancía y materia prima; en cambio sus esposos, que son pescadores, las personas que obtienen la materia prima (pescados y mariscos) los utilizan para la compra y el arreglo de sus motores fuera de borda, así como el arreglo y la confección de atarrayas y artes de pesca.

\section{Impacto económico de los microcréditos en el sector rural}

De acuerdo con Consultive Group to Assist the Poorest (CGAP) (2003), en México, el impacto económico de los microcréditos entre las mujeres rurales de escasos recursos es alto, y con la finalidad de capitalizar este sector, el FOMMUR ha otorgado desde el año 2001 a septiembre de 2012, un importe total por concepto de financiamiento de $\$ 5,117.8$ millones de pesos (Cruz Ramirez et al., 2013). Así mismo, el número de personas beneficiadas fue de 1’474.858 mujeres, a través de 1'958,537 microcréditos (Gobierno de México, 2012).

En la costa de Chiapas, la cantidad de préstamos (en miles de pesos) y el número de microcréditos que han sido otorgados a los hombres y mujeres en Arriaga, Tonalá y Pijijiapan, ciudades que integran la zona Istmo-Costa de Chiapas, durante el año 2016, se muestran en la Tabla 1.

De los microcréditos otorgados, los porcentajes utilizados como capital de trabajo y activo fijo durante el año 2016 (ver Tabla 2), puede verse que hay un elevado porcentaje (más del 60 \%) de los préstamos que se utilizan para la compra de mercancía y materia prima para los pequeños negocios que emprenden; y un porcentaje menor (del $40 \%$ ) se utiliza para compra de materiales, equipo y maquinaria. 
Tabla 1. Cantidad de préstamos (en miles de pesos) y número de microcréditos que han sido otorgados a los hombres y mujeres en la zona Istmo-Costa de Chiapas durante el año 2016

Table 1. Number of loans (in thousands of Mexican pesos) and microcredits granted to men and women in the area of the isthmus-coast of Chiapas in 2016

\begin{tabular}{lccc}
\hline Área o lugar & $\begin{array}{c}\text { Mujeres (número de } \\
\text { créditos) }\end{array}$ & $\begin{array}{c}\text { Hombres (número de } \\
\text { créditos) }\end{array}$ & $\begin{array}{c}\text { Cantidad de } \\
\text { Préstamos }\end{array}$ \\
\hline Arriaga & 7,863 & 3,332 & 2.63 \\
\hline Tonalá & 8,921 & 2,716 & 9.14 \\
\hline Pijijiapan* & 16,023 & 11,216 & 15.30 \\
\hline Total & 32,807 & 17,264 & 27.07 \\
\hline \multicolumn{2}{r}{ Fuente: elaboración propia. } \\
& *Incluye a la ciudad de Mapastepec, dentro de la zona Istmo-Costa.
\end{tabular}

Tabla 2. Porcentaje del capital de trabajo y de activo fijo que utilizan las personas a las que fueron otorgados los micropréstamos, en la zona Istmo-Costa de Chiapas durante 2016

Table 2. Percentages of working capital and fixed assets used by borrowers of microloans in the area of the isthmus-coast of Chiapas in 2016

\begin{tabular}{lcc}
\hline Área o lugar & Capital de trabajo (número de créditos) & Activo fijo (número de créditos) \\
\hline Arriaga & $63,4 \%$ & $36,6 \%$ \\
\hline Tonalá & $73,02 \%$ & $26,98 \%$ \\
\hline Pijijiapan* & $71,03 \%$ & $28,97 \%$ \\
\hline Total & $100 \%$ & $100 \%$ \\
\hline \multicolumn{4}{r}{ *Incluye a la ciudad de Mapastepec, dentro de la zona Istmo-Costa. }
\end{tabular}

Según la información obtenida del Banco Compartamos (2016), la inversión total en la costa de Chiapas durante el período 2015-2016 fue de $\$ 115,163$ miles de pesos, esto incluye a las ciudades desde Cintalapa hasta Huixtla, Chiapas.

\section{METOdOLOGÍA}

El presente estudio de tipo cuantitativo, de carácter exploratorio y descriptivo, se refiere al uso y obtención de las microfinanzas entre microemprendedores masculinos y femeninos, donde se pretende averiguar si realmente estos factores guardan una relación entre sí en cuanto a la importancia para el desarrollo económico de la región. Para obtener los datos cuantitativos, se formuló un instrumento de medición donde fue necesario preguntar acerca de los pequeños negocios, comprender el proceso comercial y la forma de emprenderlos, así como su relación con el micro financiamiento.

La muestra fue de 50 emprendedores: 39 mujeres y 11 hombres, quienes componen el sector de comercialización de pescados y mariscos en estos lugares. La metodología empleada fue la obtención de datos por medio de encuestas, con preguntas cerradas, donde se solicitó información relativa a las variables del caso.

Para el análisis de datos, primero se analizó la fiabilidad del instrumento a través del estadístico Alfa de Cronbach como estimador de la homogeneidad del conjunto de variables, así mismo para cada 
grupo de ítems. Para encontrar las características de los emprendedores se utilizó el software del programa SPSS Static, versión 2012, de IBM.

\section{RESULTADOS}

Los resultados indican que la mayoría de los empresarios tienen familiares que dependen de ellos; la mayoría de las mujeres respondieron que, 25 de ellas, lo que corresponde al 64.10 por ciento, que, si tienen dependientes y 14 de ellas, el 35.89 por ciento respondió que no tenían hijos o dependientes. Dentro del renglón de los hombres, 7 de ellos, el 63.63 por ciento dijo que si tenían dependientes económicos y el restante 4 de ellos dijeron que no los tenían. De acuerdo con el resultado en educación (ver Tabla 3), se tiene la siguiente información:

Tabla 3. Nivel educativo

Table 3. Educational attainment

\begin{tabular}{lcccccc}
\hline Género & \multicolumn{5}{c}{ Educación } \\
\hline & Primaria & Secundaria & Preparatoria & Técnico & Otro & Total \\
\hline Femenino & 16 & 14 & 6 & 1 & 2 & 39 \\
\hline Porcentaje de género & $41 \%$ & $35,9 \%$ & $15,4 \%$ & $2,6 \%$ & $5,1 \%$ & $100 \%$ \\
\hline Masculino & 6 & 2 & 1 & 0 & 2 & 11 \\
\hline Porcentaje de género & $54,5 \%$ & $18,2 \%$ & $9,1 \%$ & $0 \%$ & $18,2 \%$ & $100 \%$ \\
\hline Total & 22 & 16 & 7 & 1 & 4 & 50 \\
\hline & Fuente: elaboración propia. & & &
\end{tabular}

Para conocer el origen del microcrédito obtenido por los microempresarios (ver Tabla 4) se tiene:

Tabla 4. Origen del microcrédito

Table 4. Source of the microcredit

\begin{tabular}{lcccccc}
\hline Género & No adquirió & Microcrédito & Banco & Familiares & Personal & Total \\
\hline Femenino & 6 & 11 & 1 & 9 & 12 & 39 \\
\hline Porcentaje de género & $15,38 \%$ & $28,21 \%$ & $2,56 \%$ & $23,08 \%$ & $30,77 \%$ & $100 \%$ \\
\hline Masculino & 3 & 3 & 0 & 3 & 2 & 11 \\
\hline Porcentaje de género & $27,27 \%$ & $27,27 \%$ & $0 \%$ & $27,27 \%$ & $28,28 \%$ & $100 \%$ \\
\hline Total & 9 & 14 & 1 & 12 & 14 & 50 \\
\hline \multicolumn{7}{c}{ Fuente: elaboración propia. }
\end{tabular}

Respecto a la cantidad de empresarios que recurren cotidianamente a los microcréditos, los resultados indican que el $84,6 \%$ (33 mujeres) ha recurrido a ellos, y el restante, $15, \%$ ( 6 de ellas) no lo hace. En cambio, dentro de los hombres, el 72,7\% (8 hombres) han optado los microcréditos, y 3 hombres, el $27,3 \%$ no lo ha hecho.

Respecto al monto del préstamo utilizado (ver Tabla 5), se tiene: 
Tabla 5. Monto del microcrédito

Table 5. Amount of the microcredit

\begin{tabular}{lcccccc}
\hline Género & No adquirió & $<10000$ & $10000-20000$ & $21000-50000$ & $>50000$ & Total \\
\hline Femenino & 6 & 19 & 12 & 0 & 2 & 39 \\
\hline Porcentaje de género & $15,38 \%$ & $48.72 \%$ & $30.77 \%$ & $0 \%$ & $13 \%$ & $100 \%$ \\
\hline Masculino & 3 & 3 & 4 & 1 & 0 & 11 \\
\hline Porcentaje de género & $27,27 \%$ & $27,27 \%$ & $36.36 \%$ & $9.09 \%$ & $0 \%$ & $100 \%$ \\
\hline Total & 9 & 22 & 16 & 1 & 2 & 50 \\
\hline \multicolumn{7}{c}{ Fuente: elaboración propia. }
\end{tabular}

Respecto a la cuestión esencial del acceso a microcréditos, se tiene que estas personas utilizan los microcréditos para formalizarse como emprendedoras; en el grupo de mujeres, un gran porcentaje de ellas utilizan estos medios, el 84,6\% de ellas, también los hombres recurren a estos para crear sus negocios en un porcentaje similar al de las mujeres, el 72,7 \% de ellos.

Pero al mismo tiempo, estas personas recurren a diversos medios para obtener dinero, por ejemplo, el 28,21 \% de ellas recurrió a los microcréditos para establecer su negocio; el 30,77 \% recurrió a sus ahorros personales; el 23,08 \% recurrió a sus familiares para obtener dinero; en cambio, el restante $15,38 \%$ no adquirieron ningún préstamo, y solo una de ellas utilizo la banca comercial corresponde al 2,56\%. El comportamiento de los hombres es similar.

En la Tabla 5, puede verse que los montos de los microcréditos son variados. Dentro de las mujeres que hicieron prestaciones se tiene que el $48,72 \%$, prestaron más de $\$ 10,000.00$; el 30,77\% prestaron cantidades que van de $\$ 10,000.00$ a $\$ 20,000.00 ;$ solo el 5,13 \% prestó más de $\$ 50,000.00$; y el 15,38 $\%$ no hizo ningún préstamo.

La inversión ejercida por este banco ha permitido dotar a muchas personas de mecanismos de financiamiento para emprender una microempresa: mujeres y hombres se han formalizado y han creado pequeños negocios que les permite autoemplearse, principalmente mujeres rurales.

De acuerdo con Girón (2014), los microcréditos han sido un mito al ser tratados como bandera del desarrollo y como un mecanismo reductor de la pobreza, aunque tienen un alto impacto social, por lo que es muy difícil su transición al sector bancario tradicional debido a su poder económico, político y social similar a la del estado para otorgar y controlar los servicios financieros con aspecto social. A continuación, un análisis por tablas cruzadas.

\section{Edad y origen de los recursos}

Para determinar si este par de variables guardan alguna relación, se utilizó la prueba estadística de chi-cuadrado. Con un valor de $\chi^{\wedge} 2=25.113$ y un valor $p=0.000$, se concluye, por tanto, que sí están relacionadas tal y como se puede observar con la distribución de porcentajes que se presenta en la Tabla 6. En dicha Tabla se evidencia que las personas entre los 20 y 35 años se encuentran entre las opciones de no adquirir préstamos o en el caso de hacerlo, acudir a los microcréditos. En cambio, las personas que están entre los 36 y los 50, prefieren o contar con recursos propios o acudir a préstamos familiares. 
Tabla 6. Edad y origen de los recursos

Table 6. Age and source of the resources

\begin{tabular}{cllllll}
\hline & & No adquirió & Microcrédito & Familiares & Personal & Total \\
\hline Edad & $20-35$ & $17,1 \%$ & $26,8 \%$ & & $43,9 \%$ \\
\hline & $36-50$ & $4,9 \%$ & $7,3 \%$ & $29,3 \%$ & $14,6 \%$ & $56,1 \%$ \\
\hline Total & $22,0 \%$ & $34,1 \%$ & $29,3 \%$ & $14,6 \%$ & $100,0 \%$ \\
\hline \multicolumn{5}{c}{ Fuente: elaboración propia. }
\end{tabular}

\section{Género y origen de los recursos}

Al establecer una prueba de chi-cuadrado entre el par de variables «Género» y "Origen de los recursos», con un estadístico de $\chi^{\wedge} 2=2.435$ y un valor $p=0.487$, no se encuentra evidencia estadística suficiente para rechazar la hipótesis nula de independencia. Por tanto, no se puede hablar en términos de que el hecho de ser mujer indique alguna preferencia en términos del origen de los recursos. Asimismo, el hecho de ser hombre tampoco da indicios de alguna fuente en particular de recursos. La distribución de los porcentajes de la Tabla 7, se da simplemente por la composición de la muestra, en donde se tiene un mayor número de mujeres que de hombres.

Tabla 7. Edad y origen de los recursos

Table 7. Gender and source of the resources

\begin{tabular}{|c|c|c|c|c|c|c|}
\hline & & No adquirió & Microcrédito & Familiares & Personal & Total \\
\hline \multirow[t]{2}{*}{ Género } & Masculino & $33,3 \%$ & $21,4 \%$ & $25,0 \%$ & & $22,0 \%$ \\
\hline & Femenino & $66,7 \%$ & $78,6 \%$ & $75,0 \%$ & $100,0 \%$ & $78,0 \%$ \\
\hline Total & & $100,0 \%$ & $100,0 \%$ & $100,0 \%$ & $100,0 \%$ & $100,0 \%$ \\
\hline
\end{tabular}

\section{Nivel educativo y monto del crédito}

En cuanto al «Nivel educativo» y al «Monto del crédito» por parte de las personas encuestadas, se puede concluir con un estadístico de $\chi^{\wedge} 2=31.821$ y un valor $p=0.000$, que sí hay una relación de dependencia entre este par de variables. La Tabla 8 presenta los porcentajes para el cruce entre cada uno de los tres niveles considerados para cada una de las variables, en donde se evidencia que al parecer el nivel de educación determina no solo la posibilidad de acceder al crédito, sino también su cuantía: solo las personas con un nivel educativo de preparatoria pudieron acceder al crédito de mayor cuantía (10000-20000). Además, mientras las personas con un nivel educativo de primaria $(48,9 \%)$ se encuentran entre no contar con crédito $(20 \%)$ y contar con el monto mínimo $(<10000$, correspondiente a un $28,9 \%)$, los que tienen secundaria (35,6\%) se ubican entre un monto de crédito menor a 10000 (20\%) y un monto entre 10000 y 20000 (15,6\%).

Tabla 8. Educación y monto del crédito

Table 8. Educational attainment and amount of the credit

\begin{tabular}{|c|c|c|c|c|c|}
\hline & & No adquirió & $<10000$ & $10000-20000$ & Total \\
\hline \multirow[t]{3}{*}{ Educación } & Primaria & $20,0 \%$ & $28,9 \%$ & & $48,9 \%$ \\
\hline & Secundaria & & $20,0 \%$ & $15,6 \%$ & $35,6 \%$ \\
\hline & Preparatoria & & & $15,6 \%$ & $15,6 \%$ \\
\hline Total & & $20,0 \%$ & $48,9 \%$ & $31,1 \%$ & $100,0 \%$ \\
\hline
\end{tabular}




\section{Edad y monto del crédito}

La correspondiente prueba de chi-cuadrado para determinar la relación entre las variables de la «Edad» y el «Monto del crédito» dio como resultado una relación de dependencia ( $\chi^{\wedge} 2=13.190$, valor $p=0.001$ ), lo cual indica que la edad tiene alguna influencia en la cuantía del monto del crédito concedido. Por ejemplo, en la Tabla 9 se aprecia que los que pudieron acceder al monto de mayor cuantía fue el grupo etario entre los 36 y los 50 años. Asimismo, hay una marcada diferencia entre las personas que no adquirieron crédito y su edad (16,7 \% para los de 20 a 35 años y 4,8 \% para los de 36 a 50 años).

Tabla 9. Edad y origen de los recursos

Table 9. Age and amount of the credit

\begin{tabular}{ccllll}
\hline & & No adquirió & $<10000$ & $10000-20000$ & Total \\
\hline Edad & $20-35$ & $16,7 \%$ & $26,2 \%$ & & $42,9 \%$ \\
\hline & $36-50$ & $4,8 \%$ & $26,2 \%$ & $26,2 \%$ & $57,1 \%$ \\
\hline Total & $21,4 \%$ & $52,4 \%$ & $26,2 \%$ & $100,0 \%$ \\
\hline \multicolumn{5}{c}{ Fuente: elaboración propia. }
\end{tabular}

\section{DISCUSIÓN}

Se puede evidenciar que, a pesar de la alta rentabilidad de los microcréditos, que se traduce en el cobro de altas tasas de interés derivado por el uso de capital en ellos, muchas familias han logrado renovar la vida, mejorando su situación económica y conservando su empleo (Patiño, 2008), principalmente en el sector rural. Este capital es en realidad financiado por inversionistas de círculos financieros que forman parte del sistema financiero oculto, y que sustituyen el financiamiento otorgado por parte del Estado, con la propaganda de eliminar las desigualdades sociales y la lucha contra la pobreza, ante el fracaso de la Banca de Desarrollo (Girón, 2014).

Es necesario eliminar estas «debilidades» del modelo del microfinanciamiento, que permita que los microcréditos fueran la mejor opción, principalmente para aquellos pequeños empresarios o mini empresarios del sector rural, para que puedan acceder a los créditos sin pasar por las condiciones bancarias de los microcréditos ejercidas por los canales de la banca comercial tradicional (Hulme \& Mosley, 1996, pág. 141).

Según estudio de Román \& Tandazo (2017) en el caso de Ecuador, se evidencia que los microcréditos son una herramienta para la creación de empresas, de hecho, los emprendedores son el porcentaje más favorecido; cabe aclarar que en este estudio no se diferencia el género del emprendedor ni la ubicación física, sin embargo, provee de importante información para sugerir estudios posteriores en el tema.

Por otra parte, Merino (2015) sí se enfoca en el desarrollo rural y en el uso del microcrédito como medio para combatir la pobreza, sin embargo, en este trabajo, se discute acerca de los límites de la teoría de microfinanzas, en donde se dicta que el microcrédito es un factor para salir de la pobreza; por el contrario, la cuestiona partiendo de revisión de casos en una pequeña localidad del estado de Chiapas en México. 


\section{CONCLUSIONES}

Es notoria la falta de educación financiera que existe dentro de las comunidades pesqueras en de la costa de Chiapas, por lo tanto el objetivo del trabajo está ampliamente justificado, debido a que establecer el perfil que deben de tener las personas usuarias de este programa federal para que le sean otorgados y ser beneficiarios de los microcréditos es de importancia esencial, ya que en estos lugares no existen antecedentes que definan las características de estas personas que utilizan para crear pequeñas empresas de comercialización de pescados y mariscos, que es la actividad principal en la costa de Chiapas, dentro de un entorno de pobreza extrema y marginación.

De acuerdo con la tabla de Edad y Origen de los recursos, este tipo de comportamiento se puede deber a que los más jóvenes (20-35) pueden ser un poco más arriesgados y optar por el microcrédito en el caso de necesitar capital para su emprendimiento; mientras que los de más edad (36-50), al parecer son un poco más conservadores y prefieren no endeudarse con entidades bancarias, sino más bien obtener los recursos que necesitan por medio de ahorros o de préstamos por parte de familiares.

Al hacer el cruce y análisis de las variables «Edad» y «Origen de los recursos», se llega a la conclusión de que las personas más jóvenes (20-35 años) no adquirieron recursos o acudieron al microcrédito, mientras que los de mayor edad (36-50 años) prefirieron acudir a familiares y a recursos propios. Se complementan estas ideas, indicando que inclusive las personas de mayor edad (36-50 años) optaron por créditos de mayor cuantía (10000-20000). Lo anterior puede ser una explicación de la no preferencia por microcréditos por parte de este grupo etario (necesidad de mayores recursos, sin asumir el riesgo de no pago ante el sistema financiero) y de la correlación que se puede apreciar entre la cuantía del crédito por parte de los más jóvenes y la opción del microcrédito (limitaciones en cuanto al monto prestado).

Finalmente, se puede decir que el Micro financiamiento ha revolucionado el sector económico en estos últimos años, principalmente en el sector rural, ha sido un instrumento muy útil y de gran ayuda contra la pobreza, sin embargo, no ha sido posible realmente medir su impacto socioeconómico y su eficacia como palanca en el desarrollo social, pero como sostiene el grupo de mujeres emprendedoras de Paredón, Chiapas, «el problema del microfinanciamiento no llega a todas nosotras, debido a que hay que hacer círculos de amistades que sean realmente responsables (hablando de la metodología grupal que ejerce la banca Compartamos como requisito para dotarlas de microcréditos) y eso generalmente no ha sido posible; realmente es lo que impide que lleguen a más personas, lo que permitiría a muchas familias mejorar su situación y tener un respiro por la situación económica».

\section{REFERENCIAS}

Aguilar-Pinto, E., Tuñón-Pablos, E., \& Morales-Barragán, F. (2017). Microcrédito y pobreza. La experiencia del programa Microempresas Sociales de Banmujer en Chiapas. Economía, sociedad y territorio, 17(55), 809-835. https://dx.doi.org/10.22136/est2017885

Armendáriz, B., \& Morduch, J. (2011). Economía de las Microfinanzas. México: Fondo de Cultura Económica. 
Bateman, M. \& Chang, H. (2012). Microfinance and the Illusion of Development: From Hubris to Nemesis in Thirty Years. World Economic Review, (1), 13-36. Recuperado de https://ssrn.com/abstract=2385482

Banco Compartamos, S.A. (2015). Reporte Anual (2015). Reporte anual. Recuperado de https://www.compartamos.com.mx/wps/wcm/connect/2be3ee6f-a14c-457f-8cb343d08a494a18/ReporteAnual2015.pdf?MOD=AJPERES\&CONVERT_TO=urI\&CACHEID=2be3ee6 f-a14c-457f-8cb3-43d08a494a18

Banco Compartamos, S.A. (2016). Reporte Anual. Recuperado de https://www.compartamos.com.mx/wps/wcm/connect/7f24de15-c1b5-4701-86b1cb31d61ebe77/ReporteAnualBMV2016.pdf?MOD=AJPERES\&CONVERT_TO=url\&CACHEID=7f2 4de15-c1b5-4701-86b1-cb31d61ebe77

Cabezas, D. (2008). Microcrédito social y género. PORTES, revista mexicana de estudios sobre la Cuenca del Pacífico, 1(1), 235-258.

Cheston, S. \& Kunh, L. (2008). Empoderamiento de la mujer a través de las Microfinanzas. Recuperado de https://www.microfinancegateway.org/sites/default/files/mfg-es-documentoempoderamiento-de-la-mujer-a-traves-de-las-microfinanzas-2001.pdf

Consultive Group to Assist the Poorest (2003). El impacto del Microfinanciamiento. Reseñas para agencias de cooperación. Recuperado de http://www.findevgateway.org/sites/default/files/mfg-es-documento-el-impacto-delmicrofinanciamiento-7-2003.pdf

Cruz Ramirez, M. D., Perez Castañeda, M. S., \& Piedra Mayorga, M. V. (2013). La Opción de las microfinanzas para salir de la pobreza, en México. Ciencias Administrativas, (2). Recuperado de https://revistas.unlp.edu.ar/CADM/article/view/688

El Economista (2013). Chiapas en alerta: sobreendeudamiento popular. Recuperado de http://eleconomista.com.mx/columnas/columna-especial-valores/2013/09/03/chiapas-alertasobreendeudamiento-popular.

Esquivel, H. (2010). Medición del efecto de las microfinanzas en México. Revista Comercio Exterior. 60(1), 9-27. Recuperado de http://revistasacademicas.ucol.mx/index.php/portes/article/view/364/308

Girón, A. (2014). Neoliberalismo, microcréditos y empoderamiento de las mujeres. En Consejo Latinoamericano de Ciencias Sociales y Centro de Estudios de la Mujer, Feminismos para un cambio civilizatorio (pp. 139-153). Caracas.

Gobierno de México. (2012). Descripción General del FOMMUR. Recuperado de https://www.gob.mx/cms/uploads/attachment/file/39405/fommur_anexos.pdf 
Heller, L. (2010). Mujeres emprendedoras en América Latina y el Caribe: realidades, obstáculos y desafíos. Santiago de Chile: Naciones Unidas.

Hulme, D. \& Mosley, P. (1996). Finance against poverty. New York: Routledge Taylor \& Francis.

Merino, A. G. P. (2015). Microcrédito y desarrollo rural. Una mirada crítica a partir de un estudio de caso. EntreDiversidades, (5), 159-189.

Patiño, O. (2008). Microcrédito, Historia y experiencias exitosas de su implementación en América Latina. Revista Escuela de Administración de Negocios. (63). 41-58. https://doi.org/10.21158/01208160.n63.2008.442

Programa Nacional de Financiamiento al Microempresario (2013). ¿Qué es el PRONAFIM? Recuperado de http://www.pronafim.gob.mx/temp/pws1221asp

Román, M. B. M., \& Tandazo, L. A. A. (2017). Los microcréditos y su incidencia en el crecimiento económico de las mipymes. Sur Academia: Revista Académica-Investigativa del Área Jurídica Social y Administrativa, 7(1), 19-24. Recuperado de http://revistas.unl.edu.ec/index.php/suracademia/article/viewFile/475/374 